\title{
A propos de La loyauté dans les relations internationales de Josepha Laroche (dir.), Paris, l'Harmattan, 2001
}

About Josepha Laroche's (eds) Loyalty in International Relations

\section{Wolf-Dieter Eberwein}

\section{OpenEdition}

Journals

Édition électronique

URL : http://journals.openedition.org/conflits/848

DOI : $10.4000 /$ conflits.848

ISSN : $1777-5345$

Éditeur :

CCLS - Centre d'études sur les conflits lilberté et sécurité, L'Harmattan

Édition imprimée

Date de publication : 1 septembre 2002

Pagination : 217-222

ISBN : 2-7475-3432-4

ISSN : 1157-996X

Référence électronique

Wolf-Dieter Eberwein, «A propos de La loyauté dans les relations internationales de Josepha Laroche (dir.), Paris, l'Harmattan, 2001 », Cultures \& Conflits [En ligne], 47 | automne 2002, mis en ligne le 29 avril 2003, consulté le 30 mars 2021. URL : http://journals.openedition.org/conflits/848 ; DOI : https:// doi.org/10.4000/conflits.848

Ce document a été généré automatiquement le 30 mars 2021.

Creative Commons License 


\title{
A propos de La loyauté dans les relations internationales de Josepha Laroche (dir.), Paris, l'Harmattan, 2001
}

\author{
About Josepha Laroche's (eds) Loyalty in International Relations
}

\section{Wolf-Dieter Eberwein}

1 Cette publication engageait une prise de risque encouru à la fois par la Section d'Études Internationales de l'Association Française de Science Politique - ayant accueilli le colloque d'où sont issus les dix-sept articles - et par Josepha Laroche, organisatrice du colloque et directrice du livre. Le risque résidait dans l'inquiétude que suscitait le concept même de loyauté et plus précisément son utilité, car comme le dit MarieClaude Smouts dans l'avant-propos «il s'agissait au premier abord d'un concept mou » (p. 7). Néanmoins la séduction l'a emporté parce qu'il s'agissait d'une belle question. Le lecteur se voit ainsi confronté à des approches variées construites autour du concept de loyauté, en partie théoriques et conceptuelles, en partie empiriques.

La publication comporte quatre parties : la première traite de la loyauté dans la théorie des relations internationales, la deuxième se concentre sur la politique étrangère et loyauté, la troisième analyse la problématique de la loyauté dans l'économie internationaleet la quatrième, finalement, se préoccupe de la loyauté comme principe d'ordre. Dans son introduction Josepha Laroche ouvre le débat sur la loyauté comme cadre d'analyse, tandis que Guillaume Devin clôture le débat avec une conclusion. Il ne peut s'agir de conclusions définitives, car, selon G. Devin, l'ouvrage ne tranche pas clairement entre deux hypothèses: celle du cercle vertueux de la loyauté fondé sur une nécessité structurelle (p. 373) et celle du cercle vicieux en fonction des conduites imposées par les plus puissants (p. 374) et fondé sur l'hégémonie occidentale.

Introduire le concept de loyauté en relations internationales représente certainement un acte téméraire pour plusieurs raisons. La première : ce concept n'est pas commun. La seconde : il s'agit d'un concept psychologique au niveau microanalytique. Il s'agit 
d'une disposition à l'action d'individus, qui n'est pas observable en tant que telle, et non d'un comportement propre, de ce fait perceptible. La troisième : sans même avoir lu les analyses, on se pose d'emblée la question: pourquoi aurait-on besoin d'un concept additionnel pour analyser aujourd'hui les relations internationales aussi complexes soient-elles? Josepha Laroche ouvre le débat en retraçant le chemin philosophique de la loyauté. Elle considère le concept comme principe d'ordre supposant l'invention de la juridicisation et le respect d'une protection internationale des biens communs, ce qui implique un mode d'imposition de règles communes (p. 27). On pourrait à priori très vite se mettre d'accord sur la notion d'ordre, dont la loyauté serait la référence, mais ceci n'est pas du tout évident. Car, comme F. Charillon le suggère (p. 105), la loyauté peut être considérée en fonction de l'attachement à certains principes ou normes, ou alors comme engagement envers certains principes. Il favorise la dernière approche. Cette distinction se trouve, à mon avis, au cœur de ce débat qui essaye de cerner la problématique de l'ordre international et du respect de certaines normes censées se concrétiser dans le comportement des acteurs.

4 Patrick Lehingue retrace l'origine de cette notion spécifique de loyauté, un concept qu'a proposé Hirschmann et qui est sans doute à l'origine du débat. Comme il le dit luimême, la loyauté est une catégorie résiduelle chez Hirschmann, le parent pauvre de voice et exit(p. 99). Ce manque de précision a bien été détecté par Yves Bajoit dans son article de 1988 dans lequel il fait une distinction entre apathie et loyauté. Ces deux comportements ne peuvent être distingués en réalité car tous les deux se présentent comme attitude de fidélité muette (p. 81). Kuran, qui n'est pas mentionné par Patrick Lehingue, a précisé cet aspect en introduisant la notion de falsification des préférences individuelles. Il entend par là que les individus qui ne choisissent ni exit ni voice ont des préférences qu'ils démontrent par leur comportement de soutien aux dirigeants au pouvoir (préférence publique), et en s'abstenant, par exemple, d'adopter un comportement contestataire, et ce, bien qu'ils soient individuellement opposés aux dirigeants au pouvoir (préférence individuelle). Dans cette logique, tant que les coûts de cette falsification des préférences individuelles sont inférieurs aux coûts des préférences publiques, les individus restent loyaux. Ce qui rend la conception de Kuran aussi attractive c'est le fait qu'elle établit le lien entre loyauté (un terme qu'il n'utilise pas, il faut bien le dire) comme attachement ou non, et l'engagement. Le point essentiel de cette conception établit un lien direct entre la disposition à agir et l'abstention de s'engager.

5 La loyauté, dans ce sens, serait donc limitée à une relation hiérarchique, seulement cette relation est absente théoriquement dans l'univers de la politique internationale. Dans ce dernier cas, une hiérarchie peut certes être établie, mais elle n'est pas fondée sur un ordre légitime de représentation. Elle l'est en principe sur la puissance et sur la réputation d'être puissant. La loyauté, appliquée aux relations internationales, est donc une notion intéressante car elle ouvre des perspectives différentes des approches habituelles. Elle introduit une conception normative au sein de la représentation de la politique internationale, et c'est cette idée que tous les auteurs de l'ouvrage semblent partager. Il me semble que l'aspect normatif de l'ordre international est en effet une question centrale pour la discipline des relations internationales. Seulement ce constat fait, le concept de loyauté est il nécessaire, ou au moins utile pour analyser l'émergence de cet ordre normatif ? Quelle est la relation entre norme internationale et loyauté ? La loyauté, en tant que concept, permet-elle de décrire et d'expliquer le comportement des acteurs dans le système international? Le concept est-il utile pour la 
compréhension de l'ordre international au sens où non seulement il engagerait sur une analyse de l'acceptation de certaines normes, mais aussi, parce qu'il expliquerait le respect de ces normes, repérablesdans les comportements des acteurs?

6 Si Guillaume Devin a raison lorsqu'il affirme en conclusion qu'il n'existe pas une loyauté mais des loyautés (p. 65), nous avons deux options concernant la stabilité de la hiérarchie des loyautés. Ou bien il existe justement une hiérarchie stable de loyautés ce qui simplifie le débat - car cela nous permet d'expliquer pourquoi, dans un cas spécifique, nous sommes confrontés à un comportement déloyal ou bien à ce que Max Weber appelait un choix au sein une situation spécifique entre Gesinnungsethik et Handlungsethik. Le premier terme renvoyant à la décision d'observer une certaine norme sans tenir compte des conséquences (le martyre serait un cas classique), le deuxième à une décision considérant les conséquences (par exemple le choix d'accepter un chantage pour sauver la vie de sa propre famille, par exemple). Ainsi, même si une hiérarchie stable existe, nous avons à priori deux possibilités d'interprétation d'un comportement : ou bien il est déloyal ou il est loyal mais la loyauté dépend dans ce cas $\mathrm{du}$ système de référence que se donne l'individu, c'est à dire repose sur l'éthique du choix. Cette solution est donc défectueuse.

7 Si l'on admet par ailleurs qu'une hiérarchie stable des loyautés n'existe pas, le problème consiste à développer une conception plus complexe fondée sur un certain nombre de classes spécifiques déterminant ce qu'est le comportement loyal d'un acteur ou d'un groupe d'acteurs ou ce qu'est un comportement déloyal. Mais nous n'échappons pas non plus de toute façon au problème de l'éthique du choix activée dans ces cas spécifiques.

On pourrait interpréter cette argumentation comme une critique fondamentale de la pertinence du concept de loyauté. C'est faux. Une précision fructueuse, sinon novatrice, est introduite par Philippe Ryfman dans sa discussion sur les codes de conduite dans le champ humanitaire. La formule qu'il suggère est, dans le cas spécifique de l'humanitaire, de redéfinir la loyauté non seulement comme intention ou choix mais comme obligation, fondée sur un système spécifique de normes (ce qui serait dans ce cas le droit international humanitaire). Cette conception tient donc compte de la base normative du concept de loyauté, c'est-à-dire l'humanisme, et elle relie également cette disposition à un comportement inconditionnel concernant la protection et l'assistance aux victimes. Un lien comparable me semble exister entre normes et obligation dans le secteur de l'information, qu'explore Yves Poirmeur et Thomas Lindemann avec leur étude des loyautés envers le genre humain en temps de guerre ou Philippe Moreau Defarges avec son analyse de la loyauté à l'égard de l'humanité.

9 Ce lien direct entre la fondation normative d'un certain aspect des relations internationales et le comportement fondé sur des normes concrètes n'est pas donné ou n'existe qu'indirectement dans les relations économiques internationales ou dans le domaine de la politique étrangère. La défection par exemple qu'analysent Pierre Mazet (coopération transfrontalière) et Pascal Vennesson (alliances) représente un point de départ. Existe-t-il vraiment une norme qui pourrait être directement reliée à la défection? Je ne crois pas que ce soit le cas. Ce qui est le véritable problème, c'est l'identification des intérêts concrets des acteurs, des normes référentielles qui leur sont sous-jacentes et l'éthique de leur décision. Ainsi, ce qui peut être interprété comme déloyal par certains, ne l'est pas forcément pour d'autres, car nous devons accepter le simple fait qu'il existe des intérêts concurrentiels, des normes de références 
et des éthiques de décision différentes. Dans le cas de la politique étrangère ou du désarmement nous sommes confrontés au même problème. Même si nous supposons que tous les partis partagent la même loyauté envers le désarmement (concret) ou la politique étrangère (en général) ceci peut mener à un comportement qu'il sera facile de dénoncer comme déloyal. Aussi avec un tel verdict n'avons nous rien expliqué. La discussion autour de la loyauté chez Machiavel serait sans doute particulièrement pertinente pour approfondir cet aspect spécifique; de même que celle de Wormser se référant à Raymond Aron en discutant, entre autres, l'aspect du pluralisme.

Dans l'économie internationale, le lien direct entre normes et action se situe sur un autre plan. Car ici les normes sont fondées sur des intérêts qui, en partie, sont imposés par la puissance ou l'influence. Ce n'est qu'à un deuxième niveau qu'interviennent des normes de l'ordre soi-disant universel, telles que l'équité. La loyauté envers ces normes ne peut donc être présupposée, en particulier si elles sont plus ou moins imposées. Les analyses de Chevallier (corruption), Rainelli (commerce international) et Hidass (l'accord sur les ADPIC) me semblent soutenir cet argument.

11 En conclusion, tous les articles rassemblés dans ce volume illuminent des aspects centraux concernant la fondation normative du système international. Ils démontrent tous, en plus, la nécessité non seulement d'analyser de façon approfondie le rôle des normes des acteurs mais aussi, de dégager plus en détail les conditions et les facteurs qui facilitent ou empêchent un comportement conforme, au-delà de l'explication par la puissance et des intérêts se disant nationaux. Et, finalement, ils démontrent tous la nécessité de ce que Josepha Laroche, directrice de la publication, appelle la juridicisation des relations internationales.

12 Cet ouvrage contribuera certainement, en tous cas je l'espère, à l'intensification d'un débat absolument crucial pour notre discipline. Il sera certainement utile de relier ce débat à la discussion des normes en relations internationales actuellement en cours dans le monde anglo-saxon. Je pense entre autres au modèle de la diffusion des normes établi par Finnemore et Sikkink. Leur modèle de socialisation des normes est intéressant pour traiter de cet aspect spécifique de la loyauté. Ces auteurs suggèrent qu'une fois le processus de socialisation achevé, le comportement conforme à certaines normes devient automatique. Dès lors, dans cette logique, la notion de loyauté serait superflue. Hélas, cet argument théorique manque encore de démonstrations empiriques systématiques. Il serait également intéressant de relier la notion de loyauté au modèle en spirale de Thomas Risse et al. qui essaient d'expliquer comment des violations des normes dans le domaine des droits de l'homme dans certains pays peuvent être réduites sinon éliminées.

13 Josepha Laroche doit être félicitée pour avoir eu le courage de s'attaquer à une problématique aussi complexe que celle de la loyauté dans les relations internationales. En fin de compte le critère d'évaluation n'est pas donné par le fait de savoir si ellemême et les auteurs rassemblés dans son livre (tous des hommes à l'exception de Armelle le Bras-Chopard) ont donné des réponses définitives. Ce qui compte c'est que ce livre suscite des réactions susceptibles de nourrir le débat intellectuel au sein de la discipline. Et, de fait, ce livre a certainement provoqué une réflexion chez le lecteur que je suis. J'espère que ceci sera aussi le cas pour beaucoup d'autres. Ceci permettra peut-être, un jour ou l'autre, ou bien de trancher entre les deux hypothèses dégagées par Guillaume Devin ou de redéfinir le rôle de la loyauté dans les relations internationales. 


\section{RÉSUMÉS}

In this review essay, Wolf-DieterEeberwein comments on the book directed by Josepha Laroche, called La Loyauté dans les relations internationales (Paris, l'Harmattan). This collective book gathers the presentations that occurred during a conference by the International Studies Section of the French Political Science Association (Association Française de Science Politique) the 17 contributions discuss and spot the light on central aspects of the normative foundation of the international system and the judicialisation of international relations.

INDEX

Mots-clés : Relations Internationales 